\title{
EDITORIAL
}

\section{Hemodynamic Therapy for Delayed Cerebral Ischemia in SAH}

\author{
Michael N. Diringer
}

(C) 2018 Springer Science+Business Media, LLC, part of Springer Nature and Neurocritical Care Society

The featured article for this issue [1] addresses an important and understudied therapy routinely used in neurointensive care units: hemodynamic interventions targeted at improving cerebral perfusion in subarachnoid hemorrhage (SAH). Induced hypertension is recommended by recent guidelines [2, 3], yet its efficacy in improving outcome has not been established. The only randomized controlled trial, the HIMALAIA Study [4], was stopped prematurely after 41 patients were enrolled based on lack of effect on cerebral blood flow (CBF) and slow recruitment.

Engquist and colleagues make an important addition to the studies that have used surrogate physiologic endpoints such as $\mathrm{CBF}$ and cerebral oxygen delivery to assess proof of concept. They serially studied mechanically ventilated SAH patients with bedside xenon-computer tomography (XeCT) measurements of regional CBF. At onset of clinical suspicion of delayed cerebral ischemia (DCI), patients received a 5-day course of induced hypertension, hypervolemia, and hemodilution $(\mathrm{HHH})$ therapy. A XeCT study performed before start of $\mathrm{HHH}-$ therapy was compared to one done during therapy. They found an increase in regional $\mathrm{CBF}$ in the worst vascular territories from 19 to $\sim 27 \mathrm{ml} / 100 \mathrm{~g} / \mathrm{min}$ ) and a significant reduction in the proportion of regions with blood flow $<20 \mathrm{ml} / 100 \mathrm{~g} / \mathrm{min}$ from 26 to $10 \%$.

What should the reader take away from these findings? Several key factors must be considered. Is CBF a meaningful surrogate endpoint? Do the findings agree with other studies? How should the findings influence practice?

The first factor is not as straightforward as it appears at first glance. While CBF or perfusion been used as a surrogate for decades in the setting of ischemic stroke,

*Correspondence: diringerm@wustl.edu

Washington University, St. Louis, USA it is done so with the assumption that other factors that modify CBF (cerebral metabolism, arterial oxygen content and partial pressure of oxygen $\left[\mathrm{PaO}_{2}\right]$ and carbon dioxide) are stable. Of note, the limiting factor in cerebral ischemia is the balance between oxygen delivery and demand, so as long as hemoglobin concentration and $\mathrm{PaO}_{2}$ are stable, CBF directly matches oxygen delivery. If hemoglobin changes, that relationship no longer holds.

This assumption was not met in the present studyparticularly in regard to arterial oxygen content (due to hemodilution). Dhar raises this issue in his letter to the editor in this issue (https://doi.org/10.1007/s12028-0180517-9), and the authors discuss its implications for their work (https://doi.org/10.1007/s12028-018-0519-7).

The literature on this topic is modest, and most studies used CBF as an endpoint. They vary in terms of how CBF was measured, when it was measured in relation to the intervention and whether induced hypertension was used in conjunction with hemodilution or not. The results are heterogeneous; one study found that induced hypertension improved, but hemodilution worsened, cerebral oxygen delivery [5]. Similarly, another found that despite a rise in CBF, oxygen delivery fell due to hemodilution [6]. In studies of induced hypertension in isolation, one retrospective pilot study reported improved global CBF using mean transit time [7], whereas a prospective positron emission tomography study found no effect on CBF or oxygen delivery in regions with low baseline CBF [8].

Overall, the literature does not appear to support the concept that raising blood pressure produces a consistent rise in CBF or oxygen delivery. Yet, it is difficult to ignore bedside observations of patients who clearly clinically improve with higher blood pressure. In attempting to reconcile these apparently disparate findings, several potential explanations arise. CBF and oxygen delivery may be the wrong surrogate endpoint. That challenges our rationale that we are treating DCI. An alternative

\section{Springer}


explanation is that there is wide variation in patients' responses to raising blood pressure. In some patients, CBF will rise with induced hypertension, but in others, it will not. Recall that for CBF to rise with blood pressure increases, autoregulation must be disrupted [9], and this is not a universal occurrence in SAH .

In determining how and when to employ induced hypertension, it is important to consider the risks. It has recently been recognized that in addition to risks related to line placement and vasopressors, induced hypertension can induce Posterior Reversible Encephalopathy Syndrome and cause neurologic deterioration rather than improvement [10].

In the final analysis, it seems we should apply this therapy in a thoughtful and individualized manner. Clearly, at the onset of DCI, a trial of induced hypertension is reasonable and appropriate. If the patient improves, the course is clear- continue. If there is no change in a responsive patient, it seems appropriate to back off and explore alternative treatments. In those with limited neurologic exams, the answer is less clear, but prolonged extreme elevations should be avoided.

\section{Source of Support}

No funding.

Compliance with Ethical Standards

\section{Conflict of interest}

The author declares no conflicts of interest.

\section{References}

1. Engquist $\mathrm{H}$, Rostami $\mathrm{E}$, Ronne-Engstrom $\mathrm{E}$, et al. Effect of $\mathrm{HHH}$-Therapy on Regional CBF after Severe Subarachnoid Hemorrhage Studied by Bedside Xenon-Enhanced CT. Neurocrit Care. 2017. https://doi.org/10.1007/ s12028-017-0439-y.

2. Diringer MN, Bleck TP, Claude Hemphill J 3rd, et al. Critical care management of patients following aneurysmal subarachnoid hemorrhage: recommendations from the Neurocritical Care Society's Multidisciplinary Consensus Conference. Neurocrit Care. 2011;15(2):211-40.

3. Connolly ES Jr, Rabinstein AA, Carhuapoma JR, et al. Guidelines for the management of aneurysmal subarachnoid hemorrhage: a guideline for healthcare professionals from the American Heart Association/American Stroke Association. Stroke. 2012;43(6):1711-37.

4. Gathier CS, Dankbaar JW, van der Jagt M, et al. Effects of induced hypertension on cerebral perfusion in delayed cerebral ischemia after aneurysmal subarachnoid hemorrhage: A Randomized Clinical Trial. Stroke. 2015;46(11):3277-81.

5. Muench E, Horn P, Bauhuf C, et al. Effects of hypervolemia and hypertension on regional cerebral blood flow, intracranial pressure, and brain tissue oxygenation after subarachnoid hemorrhage. Crit Care Med. 2007;35(8):1844-51.

6. Ekelund A, Reinstrup P, Ryding E, et al. Effects of iso- and hypervolemic hemodilution on regional cerebral blood flow and oxygen delivery for patients with vasospasm after aneurysmal subarachnoid hemorrhage. Acta Neurochir. 2002;144:703-12.

7. Murphy A, de Oliveira Manoel AL, Macdonald RL, et al. Changes in cerebral perfusion with induced hypertension in aneurysmal subarachnoid hemorrhage: A Pilot and Feasibility Study. Neurocrit Care. 2017;27(1):3-10.

8. Diringer MN, Dhar R, Scalfani M, et al. Effect of high-dose simvastatin on cerebral blood flow and static autoregulation in subarachnoid hemorrhage. Neurocrit Care. 2016;25(1):56-63.

9. Diringer MN, Zazulia AR, Videen TO. Impact of induced hypertension on cerebral oxygen delivery in subarachnoid hemorrhage. Neurocrit Care. 2008;11(Supp):S3.

10. Muhammad S, Guresir A, Greschus S, et al. Posterior Reversible Encephalopathy Syndrome as an Overlooked Complication of Induced Hypertension for Cerebral Vasospasm: Systematic Review and Illustrative Case. Stroke. 2016;47(2):519-22 